\title{
Glycolytically impaired glial cells fuel neural metabolism via $\beta$-oxidation
}

Stefanie Schirmeier ( $\nabla$ stefanie.schirmeier@tu-dresden.de )

Technische Universität Dresden https://orcid.org/0000-0001-8431-9593

\section{Helen Hertenstein}

Technische Universität Dresden

\section{Ellen McMullen}

WWU Münster

\section{Leon Deharde}

Technische Universität Dresden

\section{Marko Brankatschk}

Technische Universität Dresden

\section{Biological Sciences - Article}

Keywords: glial cells, neural metabolism, glial $\beta$-oxidation

Posted Date: May 17th, 2021

DOl: https://doi.org/10.21203/rs.3.rs-251408/v1

License: (c) (1) This work is licensed under a Creative Commons Attribution 4.0 International License. Read Full License 


\section{Glycolytically impaired glial cells fuel neural metabolism via $\beta$-oxidation}

Helen Hertenstein ${ }^{1 *}$, Ellen McMullen ${ }^{2 *}$, Leon Deharde ${ }^{1}$, Marko Brankatschk ${ }^{3 \#}$ and Stefanie Schirmeier ${ }^{1 \#}$

1 Zoology and Animal Physiology, Institute of Zoology, Technische Universität Dresden, Dresden, Germany

2 current affiliation: Institut für Neuro- und Verhaltensbiologie, WWU Münster, Münster, Germany

3 Biotechnologisches Zentrum, Technische Universität Dresden, Dresden, Germany

* these authors contributed equally

\# correspondence to stefanie.schirmeier@tu-dresden.de (lead contact); for technical information on lipidomics: marko.brankatschk@tudresden.de 


\section{Summary}

Neuronal function is highly energy demanding and thus requires efficient and constant metabolite delivery. Like their mammalian counterparts Drosophila glia are highly glycolytic and provide lactate to fuel neuronal metabolism. However, flies are able to survive for several weeks in the absence of glial glycolysis ${ }^{1}$. Here, we study how glial cells maintain sufficient nutrient supply to neurons under conditions of carbohydrate restriction. We show that glycolytically impaired glia switch to fatty acid breakdown via $\beta$-oxidation and provide ketone bodies as an alternate neuronal fuel. Moreover, flies also rely on glial $\beta$-oxidation under starvation conditions with glial loss of $\beta$-oxidation increasing susceptibility to starvation. Further, we show that glial cells act as a metabolic sensor in the brain and can induce mobilization of peripheral energy stores to ensure brain metabolic homeostasis. In summary, our study gives pioneering evidence on the importance of glial $\beta$-oxidation and ketogenesis for brain function, and survival, under adverse conditions, like malnutrition. The glial capacity to utilize lipids as an energy source seems to be conserved from flies to humans.

\section{Introduction}

The nervous system consumes a disproportionally large amount of energy compared to its $s i z e^{2,3}$. Carbohydrates are the preferred energy source, thus, large quantities of glucose are imported from circulation ${ }^{4}$. Glucose is then metabolized glycolytically. The highest glycolytic activity has been associated with glial cells, which produce lactate to fuel neuronal oxidative metabolism ${ }^{5-8}$. This metabolic coupling of glial cells and neurons has been termed the "Astrocyte-Neuron Lactate Shuttle" (ANLS) and has been shown to be conserved across species ${ }^{1,9}$. Under optimal conditions glial cells are able to conserve energy by producing glycogen ${ }^{10}$ that can be used to fuel peaks of neuronal energy demand or bridge short periods of malnutrition ${ }^{10,11}$. But is glial glycogen the only alternative fuel used in periods of glucose deprivation?

It has been suggested that under conditions of nutrient restrictions the brain takes up ketone bodies from circulation to meet its energetic demand ${ }^{12,13}$. 
Whether the brain itself uses fatty acids (FA) to gain energy has been debated $^{14-17}$; even though major rate-limiting enzymes of $\beta$-oxidation are expressed in the nervous system ${ }^{18,19}$. Our study presents pioneering evidence of the importance of glial $\beta$-oxidation and ketogenesis for neuronal function and animal survival.

We analyze the metabolic plasticity of neurons and glia in the adult brain of Drosophila melanogaster. Glycolytically impaired glial cells indeed switch to $\beta$ oxidation, allowing sustained support of neuronal metabolism by supplying ketone bodies instead of glucose-derived lactate. In addition, we found that glial $\beta$-oxidation is essential for survival upon nutrient deprivation.

Furthermore, manipulations of glial glycolytic and FA metabolism are instructive for fat storage and mobilization in the fat body. Overall, we show that in dire nutritional conditions glial cells are metabolic sensors, regulating systemic lipid traffic to ensure optimal fatty acid provision. These fats in circulation provide fuel for glial $\beta$-oxidation, producing ketone bodies, to supply the nervous system with energy.

\section{Results}

Glycolytically impaired glial cells switch to $\beta$-oxidation to support neuronal function

Previously we showed that glial glycolysis is essential for neuronal survival in Drosophila, while neuronal glycolysis is dispensable ${ }^{1}$. Interestingly, glial glycolytic knockdown only reduces lifespan by about half ${ }^{1}$. Therefore, we speculated that glia use FA as an alternative fuel to ensure brain function and prolong survival. To analyze glial or neuronal breakdown of FA via $\beta$-oxidation, we targeted the enzymes Carnitine palmitoyltransferase 2 (CPT2, CG2107) and Mitochondrial trifunctional protein a subunit (Mtpa, CG4389). CPT2 is required to import acyl-carnitines into the mitochondria, while Mtpa possesses Enoyl-CoA hydratase and a Hydroxyacyl-CoA dehydrogenase activity and catalyzes breakdown of Acyl-CoA in the mitochondria. Knockdown of $\beta$ oxidation alone in neurons or glia of adult animals did not reduce lifespan $\left(C P T 2^{\mathrm{dsRNA}}\right.$, Cherry $^{\mathrm{dsRNA}}$ and $M \operatorname{tp}^{\mathrm{dsRNA}}{ }^{\mathrm{d}}$ Cherry $\left.{ }^{\mathrm{dsRNA}}\right)$. We used two UAS- 
dsRNA-constructs in all cases for better comparability with double knockdowns (Fig. 1A, S1A). While neuronal metabolic manipulations did not have any phenotypic consequences (Fig. S1A), simultaneous reduction of $\beta$-oxidation and pyruvate kinase (Pyk, a key enzyme of glycolysis) in adult glial cells significantly reduced lifespan compared to glycolysis knockdown alone (Fig. $1 \mathrm{~A})$, indicating that glial $\beta$-oxidation is essential upon glycolytic impairment.

\section{Starvation induces a metabolic switch in the glia}

Under starvation conditions, carbohydrate supply in the animal is limited ${ }^{20}$ and alternative substrates must be used for glial energy production. To examine the effect of carbohydrate restriction in animals with impaired glial $\beta$-oxidation, we starved adult flies and assessed their lifespan using a Drosophila activity monitor (DAM) setup. Animals with glycolytically impaired glia (Pyk ${ }^{d s R N A}$, CherrydsRNA) succumbed to starvation at the same rate as controls, indicating that starving animals do not rely on carbohydrate metabolism (Fig. 1B). In contrast, flies with knockdown of glial $\beta$-oxidation $\left(C P T 2^{d s R N A}\right.$, CherrydsRNA or Mtpa ${ }^{d s R N A}$, CherrydsRNA) were more susceptible to starvation than control animals (Fig. 1B). Moreover, loss of glial glycolysis in addition to $\beta$-oxidation did not have any added effect (Fig. 1B). Thus, the glial switch to $\beta$-oxidation occurs under physiological conditions like nutrient restriction and is then essential to protect the brain.

Loss of glial $\beta$-oxidation in addition to glycolysis accelerates neurodegeneration

Since neurodegeneration is most likely the cause of premature death when glial glycolysis is impaired ${ }^{1}$, we speculated that it might be accelerated upon additional loss of $\beta$-oxidation. To test this, we assessed activity of the animals, hypothesizing that neurodegeneration correlates with a loss in coordination and mobility (Fig. 1C,D, S1B,C). CPT2dsRNA , CherrydsRNA and MtpadsRNA, CherrydsRNA flies showed wild-typic activity (Fig. 1C,D). In contrast, we found that glial Pyk ${ }^{\text {dsRNA }}$ reduced activity in aged animals (22 days), indicating progressive decline of neuronal activity (Fig. 1C,D). This phenotype occurs earlier upon additional knockdown of glial $\beta$-oxidation. Pyk ${ }^{d s R N A}, C P T 2^{d s R N A}$ or $P y k^{d s R N A}, M_{t p} \alpha^{d s R N A}$, animals exhibited decreased mobility already after one 
week; and died latest at the age of three weeks. In contrast, respective neuronal metabolic manipulations did not result in abnormal phenotypes (Fig. S1B,C).

To confirm that glial loss of glycolysis and $\beta$-oxidation induces neurodegeneration, we analyzed brain morphology using semi-thin head sections. Neurodegenerative samples are characterized by areas with abnormal tissue organization ${ }^{1,21}$. Indeed, we detected holes in the cortical regions of glial double knockdown brains, already at two weeks of age (Fig. S2 $D, F)$, while impaired glial glycolysis alone induced signs of neurodegeneration only later (38 days, compare Fig. $\mathrm{S} 2 \mathrm{H}^{1}$ ). Inhibition of glial $\beta$-oxidation alone, or respective neuronal manipulations, did not lead to neurodegeneration (Fig. S1 D-I, S2). In conclusion, loss of glial glycolysis causes premature neurodegeneration ${ }^{1}$ that is strongly accelerated when glial $\beta$-oxidation is lost in addition. Thus, glial $\beta$-oxidation is sufficient to support neuronal survival and function at least for some time.

Glycolytically impaired glia produce ketone bodies to support neuronal metabolism

Neurons can metabolize ketone bodies derived from circulation ${ }^{22,23}$. These ketone bodies are generated via $\beta$-oxidation in the liver, e.g. upon carbohydrate restriction or hunger. We speculated that dietary restriction could force glia to produce ketone bodies from $\beta$-oxidation to fuel neurons. To test this, we performed glia specific knockdown of either Acetyl-CoA C-acetyltransferase (ACAT, CG10932, orthologue of human and mouse ACAT1), required to convert Acetyl-CoA into Acetoacetyl-CoA, or 3-Hydroxymethyl-3methylglutaryl-CoA lyase (Hmgcl, CG10399, orthologue of human and mouse $\mathrm{HMGCL}$ ), which catalyzes the cleavage of 3-Hydroxy-3-methylglutaryl-CoA to generate acetoacetate and Acetyl-CoA. The lifespan of flies with glially impaired ketogenesis and glycolysis is reduced to a level comparable to $\beta$ oxidation, glycolysis knockdowns (Fig. $2 \mathrm{~A}, 1 \mathrm{~A}$ ). To examine if progressive neurodegeneration is responsible for the early lethality of $A C A T^{d s R N A}, P y k^{d s R N A}$ and $H m g c^{d s R N A}, P y k^{d s R N A}$ animals, we assayed their mobility. We found that already young (1 week) animals with inhibited glial ketogenesis and glycolysis are less active, phenocopying double $\beta$-oxidation, glycolysis knockdown 
animals (Fig. $2 \mathrm{~B}, 1 \mathrm{C}$ ). This suggests that glia produce ketone bodies to fuel neurons in the absence of glycolytic activity.

\section{Loss of glial glycolysis induces mitochondrial functional changes}

Correct mitochondrial function is essential to provide energy; not only via the TCA-cycle and electron transport chain (ETC), but also $\beta$-oxidation. Analyzing the amounts of ATP5a, the ATPase of the ETC, in the brain revealed significant increase in all glial knockdown animals (Fig. S3 A), indicating changes in mitochondrial function. To further study functional alterations in mitochondria, we focused on mitochondrial morphology. It has been shown that the degree of fusion or fission of mitochondria reflects their metabolic state ${ }^{24}$. Mitochondrial $\beta$-oxidation is more efficient when the mitochondria are in a highly fused state ${ }^{25}$. To assess whether impairment of glial glycolysis changes the mitochondrial shape, we analyzed the mitochondrial appearance (using the mitochondrial reporter mitoGFP, Fig. 2 C). Indeed, mitochondria in glycolytically impaired glial cells show a much higher degree of fusion than in glial cells of control animals (Fig. 2 C,D). Such changes in mitochondrial morphology could result in differences in the abundance of lipids important for mitochondrial function, such as phosphatidylglycerol (PG) ${ }^{26,27}$. To measure PG levels, we analyzed the lipid profile of adult fly brains using mass spectroscopy (MS). PG yields were reduced in Pyk ${ }^{d s R N A}, C P T 2^{d s R N A}$ animals (Fig. $3 \mathrm{~B}$ ) and that the ratio between phosphatidylethanolamine (PE) and phosphatidylcholine (PC) was shifted (1.6; controls $=2.1)$. The PE/PC ratio correlates with the cellular capacity to produce energy, and its reduction along with low PG levels indicates mitochondrial dysfunction $^{26,27}$ (Fig. 3 C).Taken together, the loss of glial glycolysis and $\beta$ oxidation affects glial mitochondria supporting our hypothesis that glia switch to $\beta$-oxidation when glycolysis is impaired.

Impairment of glial metabolism induces mobilization of peripheral energy stores

$\beta$-oxidation is the breakdown of fatty acids, which are mostly stored as triacylglycerides (TAGs) in lipid droplets. Hence, higher $\beta$-oxidation rates should reduce cellular TAG stores. To test whether glial metabolic impairment changes TAG yields in brains, we measured TAG levels in lipid extracts from 
adult brains by MS (Fig $4 \mathrm{~A}, \mathrm{~B})$. As expected, inhibited glial $\beta$-oxidation resulted in increased TAG levels in the CNS (Fig. $4 \mathrm{~A}, \mathrm{~B}$ ). This confirms earlier work suggesting that glial loss of CPT2 induces TAG accumulation in the CNS ${ }^{15}$. Interestingly, glial knockdown of glycolysis produced similar results indicating either increased fatty acid absorption from circulation or sugar to fat conversion in the glia $^{28,29}$.

\section{Interorgan communication between glia and fat body}

Reduced glial metabolite turnover could initiate a feedback to the periphery ${ }^{30}$. To test whether loss of glial glycolysis changes lipid production and/or mobilization in fat body cells (a Drosophila tissue analogous to mammalian liver and white adipose tissue ${ }^{31}$ ), we analyzed complete head samples for their TAG content. Thin layer chromatography experiments using CNS and head samples showed that the TAG content of brains is negligible compared to the TAG content of head samples (Fig S3 A). Thus, TAG levels of adult head samples reflect primarily the TAG levels in the head fat body. MS data reveal an increase of saturated TAG levels in heads of animals with impaired glial glycolysis (Fig. $4 \mathrm{C}$ ), indicating that metabolic deficiency of the CNS triggers compensatory mechanisms in the periphery.

Since inhibition of glycolysis or $\beta$-oxidation in glia induces the buildup of TAG stores in fat body cells (Fig. 4C), we wondered whether lipids are mobilized and enter the circulation. In Drosophila, Lipophorin particles (Lpp) are the main systemic lipid carriers ${ }^{32,33}$. To assess the lipid load of Lpp, we separated them in a density gradient. In general, high lipid loads force lipoprotein particles to accumulate in less dense fractions of the gradient ${ }^{32,34}$. We found that in glial glycolysis, $\beta$-oxidation double knockdown animals, lipoproteins are more lipidated than in controls (Fig. $4 \mathrm{D}$ ); indicating a saturation of systemic lipid traffic. Nevertheless, our experiment reports the total lipid load of lipoproteins, but not the general amount of these carriers present in circulation. Lipoproteins have a shell formed by phospholipids $(P L)^{32}$. We used MS to quantify the amount of $\mathrm{PL}$ in hemolymph samples. Since extracting pure hemolymph is challenging, many samples were excluded due to tissue contamination (excluded by presence of significant amounts of TAGs or mitochondrial PGs ${ }^{32}$ ). 
Indeed, we found that $\mathrm{DAG} / \mathrm{PL}$ ratios are very different between all genotypes tested (Fig. S3 C,D). The fat body represents the main lipoprotein particle source and therefore, our data suggests a multi-dimensional regulation of this cell type by glia. Furthermore, our MS measurements confirmed high payload yields of lipoproteins in glycolysis, $\beta$-oxidation double knockdown animals (Fig. S3 C,E). However, the low levels of PLs point to low numbers of these lipid carriers (Fig S3 D,E). Taken together, our correlative results from both independent experimental approaches show that metabolic insufficiency of glia results in the mobilization of storage lipids. In addition, our data suggest an interorgan communication between glia and the periphery, responsible for controlling peripheral lipid storage.

\section{Discussion}

Neurons need a large amount of energy to function. This energy is provided with the help of glial cells that are glycolytically active, providing neurons with metabolites such as lactate 1,7,35. Here we show that glial metabolism is not limited to glycolysis but is flexible and can switch to the use of FA to fuel neuronal metabolism when carbohydrate metabolism is not sufficient.

It has long been thought that the brain is mostly restricted to carbohydrates as an energy source ${ }^{19}$. However, Drosophila glial cells can also metabolize lipids via $\beta$-oxidation to produce ketone bodies to fuel neuronal metabolism (Fig. $1,2,3)$. When glycolytically impaired glial cells additionally lose the capacity to perform $\beta$-oxidation, or produce ketone bodies, neurodegeneration occurs at an accelerated rate and the activity and lifespan of the animal are greatly reduced compared to loss of glycolysis alone (Fig. 1, 2). In addition, animals with a glial loss of $\beta$-oxidation are more susceptible to starvation. Thus, glial $\beta$ oxidation is essential to maintain brain energy homeostasis when carbohydrate metabolism is restricted genetically or due to hypoglycemia.

In the insect CNS, lipid droplets are found in glial cells ${ }^{15}$. These lipid droplets are most likely initially used to fuel $\beta$-oxidation, since loss of $\beta$-oxidation leads to a glial accumulation of lipid droplets ${ }^{15}$. In mammals no lipid droplets are found in glial cells, but oligodendrocytes produce large amounts of lipid-rich myelin. 
The primary function of myelin is thought to be insulation of the axons, to allow fast signal conduction. Interestingly, Asadollahi et al. (this issue) show that myelin can also be used as energy storage under conditions of carbohydrate restriction. Here, mouse oligodendrocytes use $\beta$-oxidation to maintain energy homeostasis in the optic nerve at the expense of myelin thickness. Also, oligodendrocyte-specific knockout of Glut1, limiting glucose uptake, leads to a reduction in myelin thickness in vivo. In addition, MRI scans of patients suffering from anorexia nervosa show a loss of white matter, indicating that severe nutrient restriction in humans can also cause oligodendrocytes to metabolize lipids stored in the myelin sheath, to maintain cerebral energy homeostasis ${ }^{36,37}$. Thus, the metabolic flexibility of glial cells to switch to $\beta$-oxidation is essential for maintaining energy homeostasis under restrictive conditions from Drosophila to humans.

In addition to using glia-intrinsic lipid stores, Drosophila glial cells can signal to the periphery to mobilize organismal energy stores to maintain adequate energy supply to neurons (Fig. 4). This indicates a new route of interorgan communication, used to maintain energy homeostasis in the brain. Also this interorgan communication is likely to be conserved, since loss of cerebral $\beta$ oxidation in mammals induces changes in peripheral metabolism ${ }^{17}$. Thus, we conclude, glial cells are a metabolic sensor regulating systemic lipid mobilization. Future studies are needed to decipher the regulatory mechanisms that govern this novel interorgan communication.

\section{Acknowledgements}

We thank Cornelia Maas for help with TLCs, Susanne Broschk for help with the LD gradients and Astrid Fleige and Sebastian Goertz for help with semi-thin sections. We thank Lipotype GmbH (Dresden, Germany) for MS analysis. This work was supported by grants from the DFG to M.B. (BR5490/2-1 and FOR2682-TP4) and S.S. (SCHI 1380/2-1). 


\section{Author contributions}

$\mathrm{HH}$ and EM designed and conducted most experiments. Lipidomic experiments were designed by $\mathrm{MB}$ and conducted by $\mathrm{HH}$, EM and $\mathrm{MB}$. LD analyzed mitochondrial morphology. SS conceived the study, assisted in designing and interpreting experiments and wrote the manuscript with the help of $\mathrm{HH}, \mathrm{EM}$ and MB. SS and MB obtained funding.

\section{References}

1. Volkenhoff, A. et al. Glial Glycolysis Is Essential for Neuronal Survival in Drosophila. Cell Metab. 22, 437-447 (2015).

2. Laughlin, S. B., de Ruyter Van Steveninck, R. R. \& Anderson, J. C. The metabolic cost of neural information. Nat. Neurosci. 1, 36-41 (1998).

3. Harris, J. J., Jolivet, R. \& Attwell, D. Synaptic energy use and supply. Neuron 75, 762-777 (2012).

4. Kuzawa, C. W. et al. Metabolic costs and evolutionary implications of human brain development. Proc. Natl. Acad. Sci. U. S. A. 111, 1301013015 (2014).

5. Pellerin, L. \& Magistretti, P. J. Glutamate uptake into astrocytes stimulates aerobic glycolysis: a mechanism coupling neuronal activity to glucose utilization. Proc. Natl. Acad. Sci. U. S. A. 91, 10625-10629 (1994).

6. Hall, C. N., Klein-Flugge, M. C., Howarth, C. \& Attwell, D. Oxidative Phosphorylation, Not Glycolysis, Powers Presynaptic and Postsynaptic Mechanisms Underlying Brain Information Processing. J. Neurosci. 32, 8940-8951 (2012).

7. Fünfschilling, U. et al. Glycolytic oligodendrocytes maintain myelin and long-term axonal integrity. Nature 485, 517-521 (2012).

8. Lee, Y. et al. Oligodendroglia metabolically support axons and contribute to neurodegeneration. Nature 487, 443-448 (2012).

9. Tsacopoulos, M., Veuthey, A. L., Saravelos, S. G., Perrottet, P. \& Tsoupras, G. Glial Cells Transform Glucose to Alanine, which Fuels the Neurons in the Honeybee Retina. J. Neurosci. 14, 1339-1351 (1994). 
10. Waitt, A. E., Reed, L., Ransom, B. R. \& Brown, A. M. Emerging roles for glycogen in the CNS. Frontiers in Molecular Neuroscience (2017). doi:10.3389/fnmol.2017.00073

11. Brown, A. M. \& Ransom, B. R. Astrocyte glycogen as an emergency fuel under conditions of glucose deprivation or intense neural activity. Metab. Brain Dis. (2014). doi:10.1007/s11011-014-9588-2

12. Hawkins, R. A., Williamson, D. H. \& Krebs, H. A. Ketone-body utilization by adult and suckling rat brain in vivo. Biochem. J. (1971). doi:10.1042/bj1220013

13. Owen, O. E. et al. Brain metabolism during fasting. J. Clin. Invest. (1967). doi:10.1172/JCI105650

14. Ebert, D., Haller, R. G. \& Walton, M. E. Energy contribution of octanoate to intact rat brain metabolism measured by $13 \mathrm{C}$ nuclear magnetic resonance spectroscopy. J. Neurosci. 23, 5928-35 (2003).

15. Schulz, J. G. et al. Glial $\beta$-oxidation regulates Drosophila energy metabolism. Sci. Rep. 5, 7805 (2015).

16. Sandra Chocron, E. et al. The trifunctional protein mediates thyroid hormone receptor-dependent stimulation of mitochondria metabolism. Mol. Endocrinol. (2012). doi:10.1210/me.2011-1348

17. White, C. J. et al. Determining the Bioenergetic Capacity for Fatty Acid Oxidation in the Mammalian Nervous System. Mol. Cell. Biol. (2020). doi:10.1128/mcb.00037-20

18. Lavrentyev, E. N., Matta, S. G. \& Cook, G. A. Expression of three carnitine palmitoyltransferase-I isoforms in 10 regions of the rat brain during feeding, fasting, and diabetes. Biochem. Biophys. Res. Commun. (2004). doi:10.1016/j.bbrc.2004.01.040

19. Clarke, D. \& Sokoloff, L. Circulation and Energy Metabolism of the Brain. Basic Neurochemistry: Molecular, Cellular and Medical Aspects. (1999).

20. Wilinski, D. et al. Rapid metabolic shifts occur during the transition between hunger and satiety in Drosophila melanogaster. Nat. Commun. 10, 4052 (2019).

21. Kretzschmar, D., Hasan, G., Sharma, S., Heisenberg, M. \& Benzer, S. The swiss cheese mutant causes glial hyperwrapping and brain 
degeneration in Drosophila. J. Neurosci. 17, 7425-7432 (1997).

22. Robinson, A. M. \& Williamson, D. H. Physiological roles of ketone bodies as substrates and signals in mammalian tissues. Physiological Reviews (1980). doi:10.1152/physrev.1980.60.1.143

23. Edmond, J., Auestad, N., Robbins, R. A. \& Bergstrom, J. D. Ketone body metabolism in the neonate: development and the effect of diet. Federation Proceedings (1985).

24. Hoppins, S. The regulation of mitochondrial dynamics. Curr. Opin. Cell Biol. 29, 46-52 (2014).

25. Rambold, A. S., Cohen, S. \& Lippincott-Schwartz, J. Fatty Acid Trafficking in Starved Cells: Regulation by Lipid Droplet Lipolysis, Autophagy, and Mitochondrial Fusion Dynamics. Dev. Cell 32, 678-692 (2015).

26. Basu Ball, W., Neff, J. K. \& Gohil, V. M. The role of nonbilayer phospholipids in mitochondrial structure and function. FEBS Letters (2018). doi:10.1002/1873-3468.12887

27. van der Veen, J. N. et al. The critical role of phosphatidylcholine and phosphatidylethanolamine metabolism in health and disease.

Biochimica et Biophysica Acta - Biomembranes (2017). doi:10.1016/j.bbamem.2017.04.006

28. Cabirol-Pol, M.-J., Khalil, B., Rival, T., Faivre-Sarrailh, C. \& Besson, M. T. Glial lipid droplets and neurodegeneration in a Drosophila model of complex I deficiency. Glia n/a--n/a (2017). doi:10.1002/glia.23290

29. Liu, L. et al. Glial Lipid Droplets and ROS Induced by Mitochondrial Defects Promote Neurodegeneration. Cell 160, 177-190 (2015).

30. García-Cáceres, C. et al. Role of astrocytes, microglia, and tanycytes in brain control of systemic metabolism. Nat. Neurosci. 22, 7-14 (2019).

31. Arrese, E. L. \& Soulages, J. L. Insect fat body: energy, metabolism, and regulation. Annu. Rev. Entomol. 55, 207-25 (2010).

32. Palm, W. et al. Lipoproteins in Drosophila melanogaster--assembly, function, and influence on tissue lipid composition. PLOS Genet. $\mathbf{8}$, e1002828 (2012).

33. Carvalho, M. et al. Effects of diet and development on the Drosophila lipidome. Mol. Syst. Biol. (2012). doi:10.1038/msb.2012.29 
34. Brankatschk, M. \& Eaton, S. Lipoprotein Particles Cross the Blood-Brain Barrier in Drosophila. J. Neurosci. 30, 10441-10447 (2010).

35. Pellerin, L. \& Magistretti, P. J. Sweet sixteen for ANLS. J. Cereb. Blood Flow \&amp; Metab. 32, 1152-1166 (2012).

36. Swayze, V. W. 2nd et al. Brain tissue volume segmentation in patients with anorexia nervosa before and after weight normalization. Int. J. Eat. Disord. 33, 33-44 (2003).

37. Nickel, K. et al. White Matter Abnormalities in the Corpus Callosum in Acute and Recovered Anorexia Nervosa Patients-A Diffusion Tensor Imaging Study. Front. psychiatry 10, 490 (2019).

38. Awasaki, T., Lai, S. L., Ito, K. \& Lee, T. Organization and Postembryonic Development of Glial Cells in the Adult Central Brain of Drosophila. J. Neurosci. 28, 13742-13753 (2008).

39. McGuire, S. E., Le, P. T., Osborn, A. J., Matsumoto, K. \& Davis, R. L. Spatiotemporal rescue of memory dysfunction in Drosophila. Science 302, 1765-1768 (2003).

40. Lin, D. M. \& Goodman, C. S. Ectopic and increased expression of fasciclin II alters motoneuron growth cone guidance. Neuron 13, 507523 (1994).

41. Lee, B. P. \& Jones, B. W. Transcriptional regulation of the Drosophila glial gene repo. Mech. Dev. 122, 849-862 (2005).

42. Sepp, K. J. \& Auld, V. J. Conversion of lacZ enhancer trap lines to GAL4 lines using targeted transposition in Drosophila melanogaster. Genetics 151, 1093-1101 (1999).

43. Steiger, D., Furrer, M., Schwinkendorf, D. \& Gallant, P. Maxindependent functions of Myc in Drosophila melanogaster. Nat. Genet. 40, 1084-1091 (2008).

44. Stork, T. et al. Organization and Function of the Blood Brain Barrier in Drosophila. J. Neurosci. 28, 587-597 (2008).

45. Wessel, D. \& Flügge, U. I. A method for the quantitative recovery of protein in dilute solution in the presence of detergents and lipids. Anal. Biochem. 138, 141-143 (1984).

46. Zobel, T. et al. Cooperative functions of the two F-BAR proteins Cip4 and Nostrin in the regulation of E-cadherin in epithelial morphogenesis. 
J. Cell Sci. 128, 499 LP - 515 (2015).

47. Ejsing, C. S. et al. Global analysis of the yeast lipidome by quantitative shotgun mass spectrometry. Proc. Natl. Acad. Sci. U. S. A. 106, 21362141 (2009).

48. Klose, C. et al. Flexibility of a eukaryotic lipidome - insights from yeast lipidomics. PLoS One 7, (2012).

49. Herzog, R. et al. Lipidxplorer: A software for consensual cross-platform lipidomics. PLoS One 7, 15-20 (2012).

50. Herzog, R. et al. A novel informatics concept for high-throughput shotgun lipidomics based on the molecular fragmentation query language. Genome Biol. 12, 1-25 (2011).

51. Trautenberg, L. C. et al. Selective Phosphorylation of Akt/ProteinKinase B Isoforms in Response to Dietary Cues. Front. cell Dev. Biol. 7, 206 (2019).

52. Mohammad, A., Moheman, A. \& El-Desoky, G. Amino acid and vitamin determinations by TLC/HPTLC: review of the current state. Open Chem. 10, 731-750 (2012).

53. Löfgren, L. et al. The BUME method: a novel automated chloroform-free 96-well total lipid extraction method for blood plasma. J. Lipid Res. 53, 1690-1700 (2012). 


\section{Figures}

\section{Figure 1}
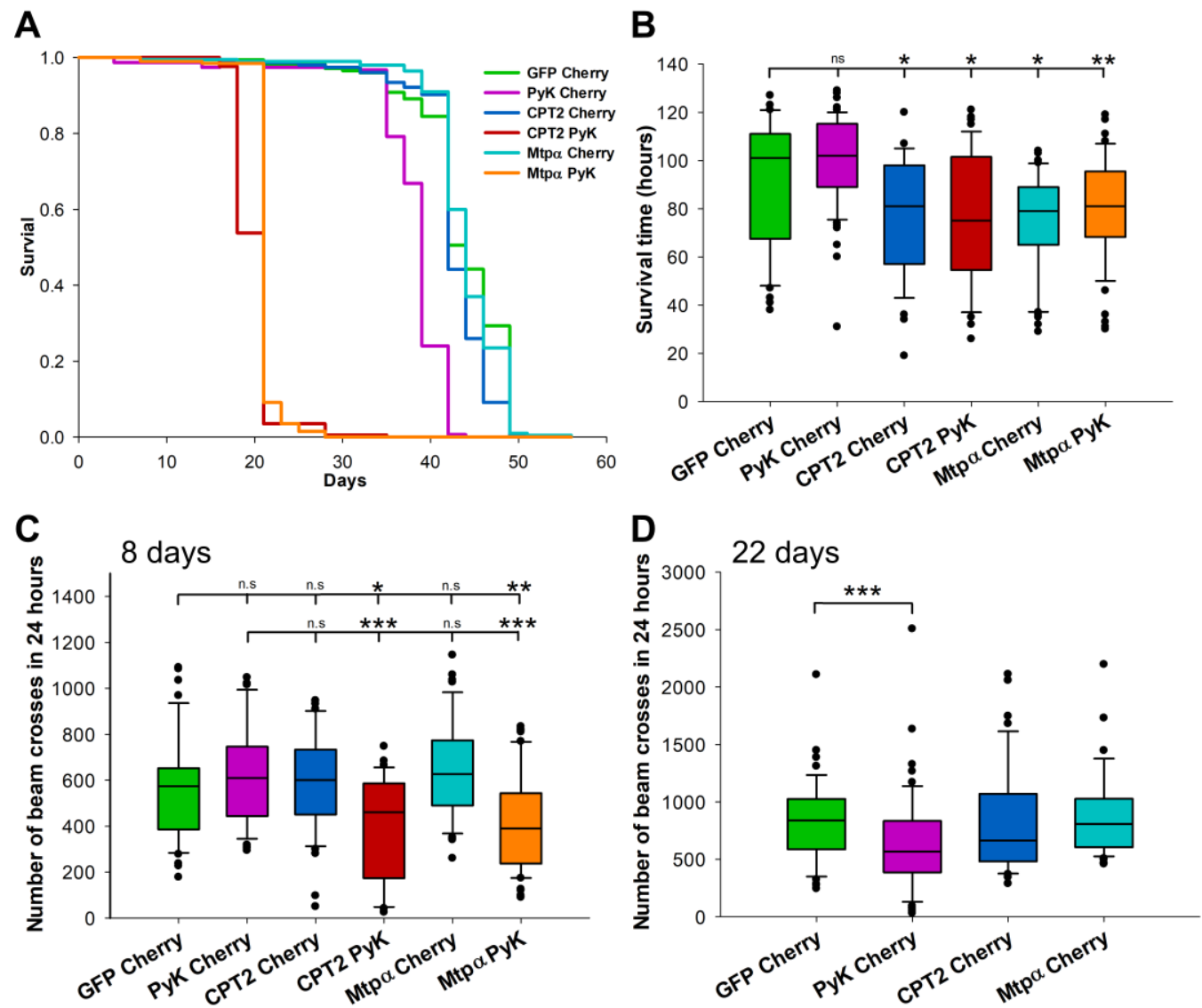

Figure 1: Glia rely on $\beta$-oxidation upon loss of glycolysis or nutrient restriction

A) Lifespan of glial glycolysis/ $\beta$-oxidation knockdown animals (CPT2 ${ }^{\text {dsRNA }}$, Pyk $^{\text {dsRnA }}$ and Mtpa ${ }^{\text {dsRNA }}$, Pyk ${ }^{d s R N A}$ ) is significantly reduced compared to glycolysis only knockdown (Pyk, Cherry). $\mathrm{N}=3, \mathrm{n} \geq 125$ B) In contrast to glycolysis loss, $\beta$-oxidation knockdown induces starvation susceptibility. $N=2$, $\mathrm{n}=50-64 \mathrm{C}$ ) Locomotive activity is reduced in glycolysis/ $\beta$-oxidation knockdown animals at the age of one week. $N=3, n \geq 60$ D) By three weeks of age the activity of glial glycolysis knockdowns is significantly reduced. $\beta$-oxidation knockdown animals (CPT2 ${ }^{\text {dsRNA }}$, Cherry dsRNA and Mtpa ${ }^{\text {dsRNA }}$, CherrydsRNA $)$ show wild-typic activity. $\mathrm{N}=3, \mathrm{n} \geq 60$. 


\section{Figure 2}

A



C



B
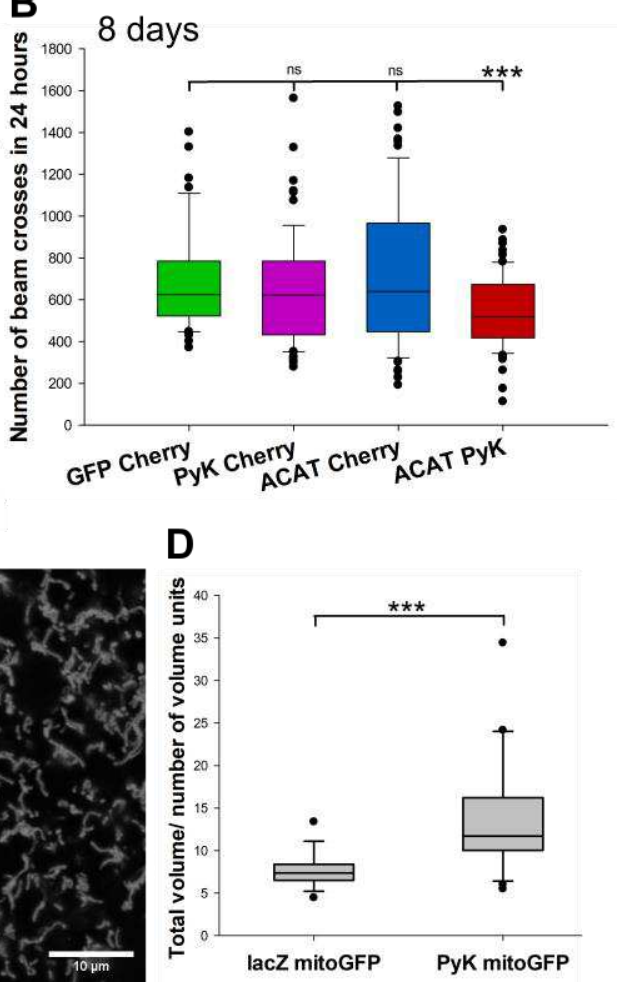

Figure 2: Glycolytically impaired glial cells produce ketone bodies via mitochondrial $\beta$-oxidation.

A) Simultaneous knockdown of ketone body synthesis (ACATdsRNA or $\mathrm{HmgcldsRNA}^{\mathrm{d}}$ ) and glycolysis (Pyk ${ }^{\mathrm{dsRNA}}$ ) in adult glia reduces lifespan compared to glycolysis only knockdowns. Ketone body synthesis only knockdown animals show wild-typic lifespan. B) Average locomotive activity is significantly decreased in one week old Pyk ${ }^{\text {dsRNA }}$, ACAT ${ }^{\text {dsRNA }}$ double knockdown animals. C) Upon glial loss of glycolysis ( $\mathrm{Pyk}^{\mathrm{ds} R N A}$ ), the mitochondria are in a more fused state then in control animals (lacZ ${ }^{\text {dsRAA }}$ ). D) Total mitochondrial volume per volume units. $\mathrm{N}=3, \mathrm{n} \geq 18$. 


\section{Figure 3}

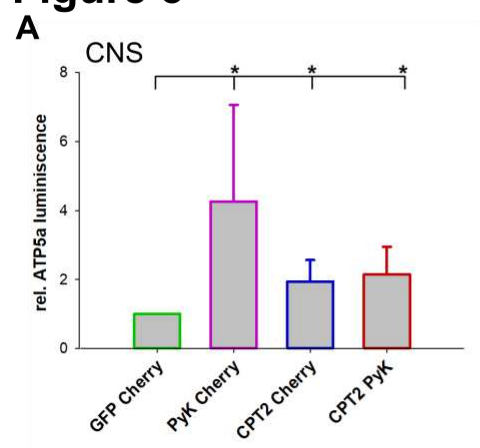

B

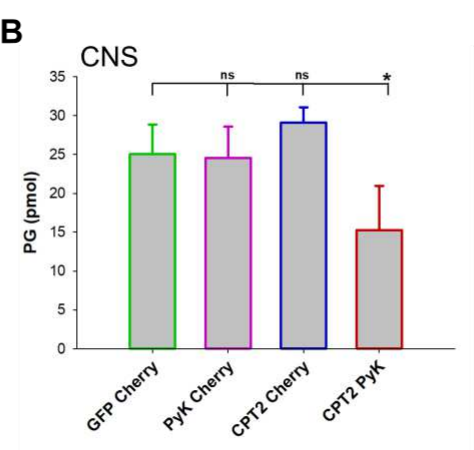

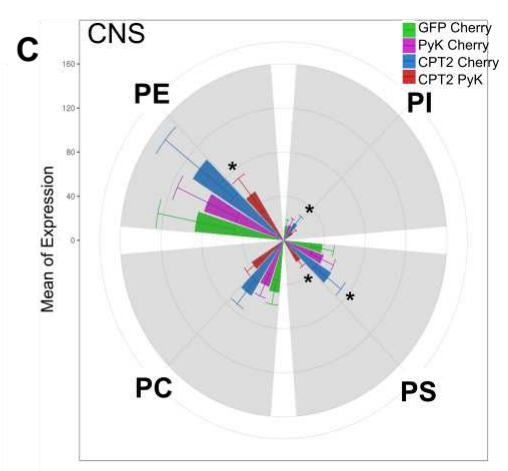

Figure 3: Glial metabolic impairment alters membrane composition in the CNS

A) Quantification of relative luminescence of ATP5 $\alpha$ normalized to protein levels. All genotypes show a significant increase in ATP5 $\alpha$ compared to controls. $\mathrm{N}=2 \mathrm{~B}$ ) Glial glycolysis, $\beta$-oxidation double knockdown (CPT2 PyKdsRNA) significantly reduces the amount of PGs detected in the CNS. $N=3 \mathrm{C}$ ) Levels of PE, PI, PS and PC in the CNS. $\beta$-oxidation only knockdown increases membrane lipid levels, while simultaneous loss of $\beta$-oxidation and glycolysis in glia reduces membrane phospholipid concentrations. $\mathrm{N}=3$. Phosphatidylinositol (PI), Phosphatidylethanolamine (PE), Phosphatidylcholine (PC), Phosphatidylserine (PS), Phosphatidylglycerol (PG). 
Figure 4

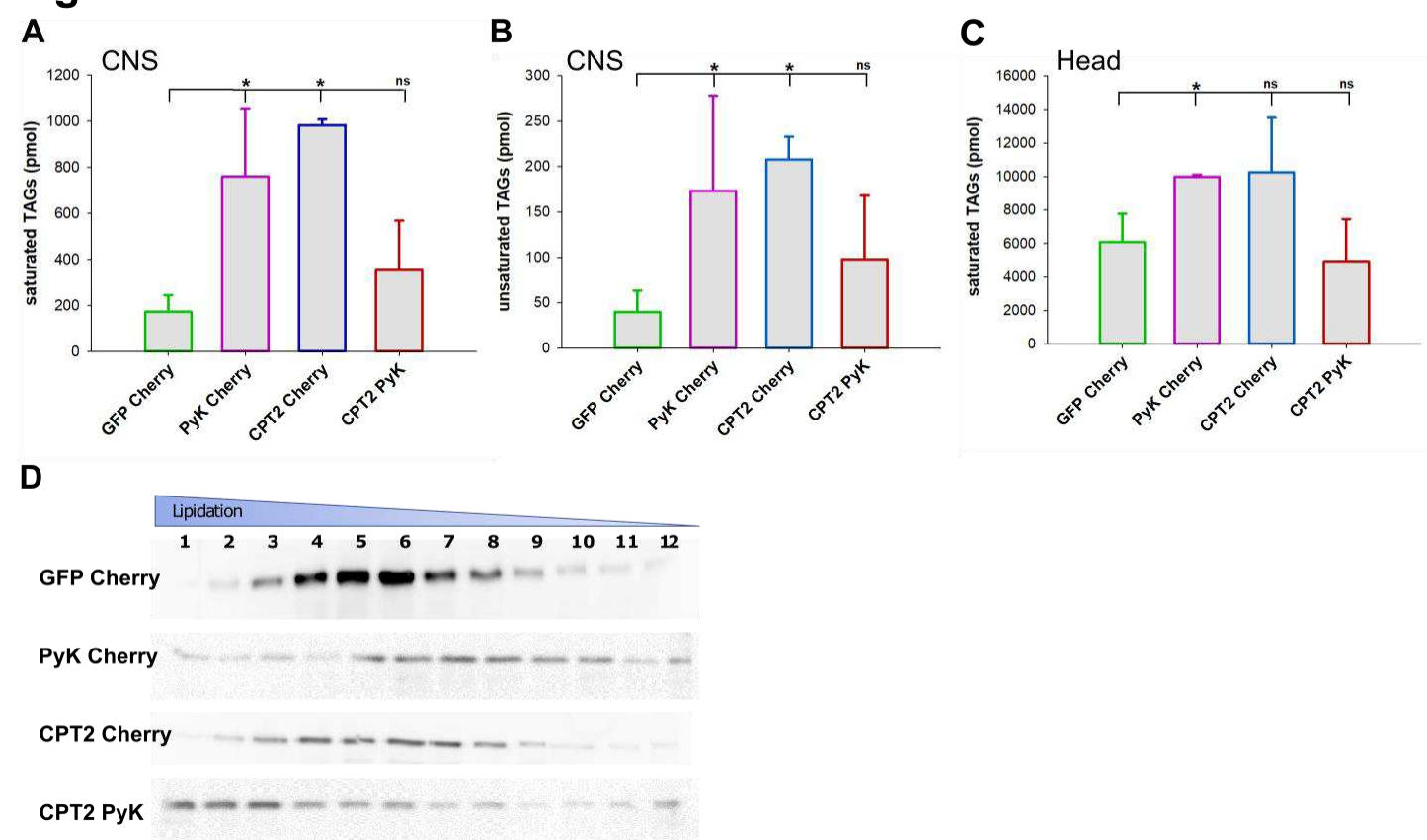

Figure 4: Impaired glia metabolism affects lipid homeostasis

$A, B$ ) Levels of saturated (A) and unsaturated (B) TAGs in the CNS are significantly increased upon glial glycolysis (Pyk ${ }^{\mathrm{dsRNA}}$ ) or $\beta$-oxidation (CPT2 ${ }^{\text {dsRNA }}$ ) knockdown. C) Yields of saturated TAGs in total head samples are significantly increased upon glial glycolytic impairment. D) Exemplary photographs from experiments depicting the Lpp signal distribution in different fractions of density gradients. Fraction 1 (left) represents sample with lowest density and Fraction 12 (right) the sample with the highest density. 


\section{Methods}

Fly stocks

Flies were maintained at room temperature on standard food. Fly crosses were kept at $18^{\circ} \mathrm{C}$ for 4 weeks on standard food. After hatching, mated female progeny of the correct genotype were transferred to $29^{\circ} \mathrm{C}$ and flipped onto new food every second day.

repoGal80 ${ }^{38}$, tubGal80ts ${ }^{39}$, elavGal4 ${ }^{40}$, repoGal4 ${ }^{41,42}$, lacZ ${ }^{\text {dsRNA }}{ }^{43}$; Flies obtained from Bloomington Drosophila stock center: elavGal4 (BL8765, BL8760), tubGal80ts (BL7017), UAS-mito-GFP (BL8443), CherrydsRNA (BL35785), GFPdsRNA (BL9331), CPT2HMJ23935 (BL62455), ACATHMC03340(BL51785), Hmgc/HMC03435 (BL 5186); Flies obtained from VDRC : PyKGD12123, MTPaGD11299

\section{Lifespan analysis}

Mated females were collected in vials containing 20 flies and kept at $29^{\circ} \mathrm{C}$ for the duration of the experiment. Vials were flipped three times per week onto fresh food, deaths were recorded, and survival rates were calculated using logrank test.

\section{Starvation}

Mated females were raised on standard food for one week at $29{ }^{\circ} \mathrm{C}$, then transferred to individual capillaries containing plain agar. Capillaries were placed in a Drosophila activity monitor with a 12-hour light dark cycle at $29^{\circ} \mathrm{C}$. Flies were counted as dead once movement ceased.

Drosophila activity monitoring (DAM)

Mated females aged for either one or three weeks at $29^{\circ} \mathrm{C}$ on standard food were loaded into individual capillaries containing $5 \%$ sucrose in agar. Capillaries were placed in a Drosophila activity monitor with a 12-hour light dark cycle at $29^{\circ} \mathrm{C}$. The activity of the flies over a 24-hour period was recorded and difference in activity was determined using Mann-Whitney rank sum test. 


\section{Semi-thin epon sections}

Heads of adult flies, aged for 17 or 38 days at $29^{\circ} \mathrm{C}$, were embedded in Epon as described previously ${ }^{44}$. $1 \mu \mathrm{m}$ semi-thin sections were cut using a EM UC7 microtome (Leica), stained with toluidine blue and imaged using a Zeiss Axiophot.

\section{Analysis of mitochondrial shape}

Brains of 17 day old animals raised at $29{ }^{\circ} \mathrm{C}$ were dissected and fixed in $4 \%$ paraformaldehyde in PBS for 1.5h at RT. Images were acquired using a Leica TCS SP8 confocal laser scanning microscope, using the objective HC PL APO CS2 40x/1.30 OIL, numerical aperture 1.3, pinhole $999.61 \mathrm{mAU}, 600 \mathrm{~Hz}$, $2048 \times 2048$ resolution and $z$ slice distance of $0.3 \mu \mathrm{m}$. $30 \mu \mathrm{m}$ stacks were scanned. Images were 3D reconstructed using Bitplane Imaris. An area of $512 \times 512$ pixels was selected, reconstruction was carried out for $13,5 \mu \mathrm{m}$ stacks starting at $7,5 \mu \mathrm{m}$ and ending at $21 \mu \mathrm{m}$ of respective $30 \mu \mathrm{m}$ stacks. Parameters used: smoothening active and set to 0.189 , local background subtraction active and set to $0.5 \mu \mathrm{m}$, threshold set to 4 . The volume data (total volume and volume units) was extracted and quantified. Wilcoxon rank sum test was used for statistical analysis.

\section{Hemolymph extraction}

30 flies after kept for 16 days on $29^{\circ} \mathrm{C}$ before being frozen at $-20^{\circ} \mathrm{C}$ for at least $4 \mathrm{~h}$ in TLC-buffer. After thawing hemolymph was extracted from flies by centrifugation (500 g, RT, $1 \mathrm{~min}$ ).

\section{Isopycnic gradient}

An isopycnic gradient was achieved by centrifuging hemolymph samples in $0.5 \mathrm{~g} / \mathrm{ml} \mathrm{KBr}$ solution for $16 \mathrm{~h}$ at $4^{\circ} \mathrm{C}, 15000 \mathrm{~g}$ in vacuum. 12 fractions were taken from each sample and proteins were precipitated by the methanol/chloroform technique ${ }^{45}$ 
SDS-PAGE and Western blotting analysis:

SDS Page and western blotting was performed following published protocols: Analyzing mitochondria ${ }^{46}$ using a-ATP5a (Abcam) and HRP (Thermo Fisher Scientific). Brain lysates were generated from 16 days old adult brains.

\section{Protein estimation}

Total protein content was examined following the manufacturer's manual (BCA Kit, Pierce).

Mass spectrometry analysis of lipidome

Mass spectrometry-based lipid analysis was performed from CNS samples, head samples or hemolymph samples by Lipotype $\mathrm{GmbH}$ (Dresden, Germany) using Lipotype Shotgun Lipidomics technology $47,48.5 \mathrm{CNS}, 5$ fly heads or 30 flies (all after 16 days at $29^{\circ} \mathrm{C}$ ) were collected ant put into ice cold TLC-buffer and frozen at $-80{ }^{\circ} \mathrm{C}$ or $-20{ }^{\circ} \mathrm{C}$. Lipids were extracted by the two-step chloroform/methanol procedure ${ }^{47}$. Data were analyzed by Lipotype $\mathrm{GmbH}$ using their lipid identification software based on LipidXplorer ${ }^{49,50}$. For raw data see supplementary files 1-3.

\section{Thin-layer chromatography}

Samples were prepared as in $\mathrm{in}^{51,52}$ and lipids extracted by the BUME method ${ }^{53}$. Extracted lipids were stored in chloroform/methanol $(2: 1)$ solution at $-80^{\circ} \mathrm{C}$.

\section{Statistics}

Statistical analysis was performed using Sigma Plot. If data was normally distributed t-test was performed. For not normally distributed data rank sum test was used. ns=not significant, ${ }^{*} p \leq 0.05,{ }^{* *} p \leq 0.01,{ }^{* * *} p \leq 0.001$. 


\section{Extended data:}

\section{Extended Data figure legends:}

\section{Figure S1}


Figure S1: Neuronal knockdown of glycolysis or $\beta$-oxidation induces no phenotypic consequences A) Animals with neuronal suppression of glycolysis and/or $\beta$-oxidation show wild-typic lifespan. $N=3, n \geq 125$. B \& C) Neuronal glycolysis and/or $\beta$-oxidation knockdown animals show wild-typic locomotive activity. $N=3, n \geq 60$. D-I) Histological brain semi-thin sections of neuronal glycolysis and/or $\beta$-oxidation knockdown animals show no signs of neurodegeneration (age of 38 days). 
Figure S2
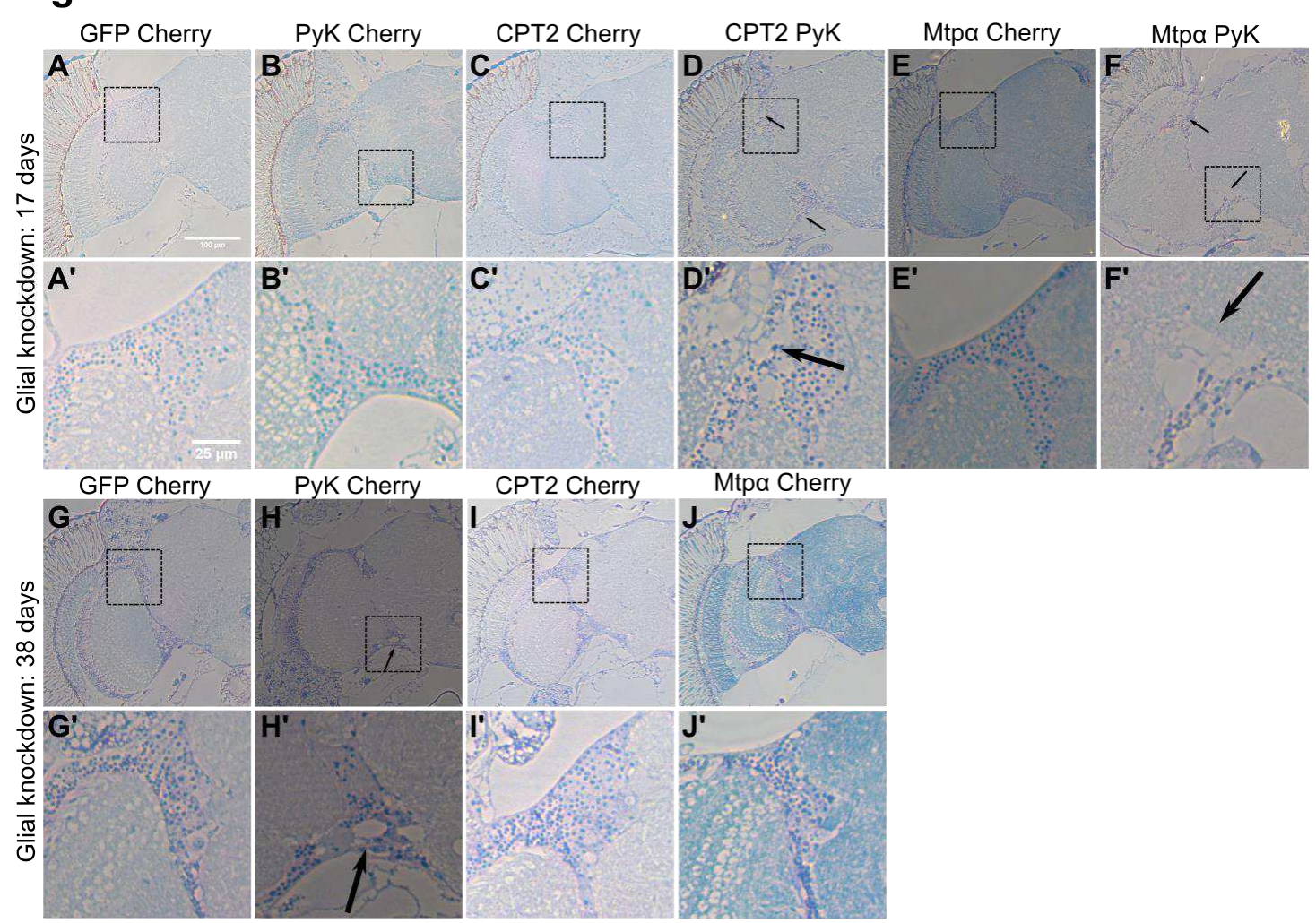

Figure S2: Loss of glial $\beta$-oxidation in addition to loss of glycolysis accelerates neurodegeneration. (A-J) Semi-thin sections (one hemisphere) of adult heads. (A' - J') Close ups of cortex area in A-J (rectangle). (A-J) Semithin sections of 17-day old animals of the indicated genotypes. (G-J) Semi-thin sections of 38-day old animals of the indicated genotypes. Animals with simultaneous knockdown of $\beta$-oxidation and glycolysis in glia (CPT2 ${ }^{\text {dsRNA, }}$ $P y k^{d s R N A}$ or MTPadsRNA, Pyk ${ }^{d s R N A}$ ) show severe signs of neurodegeneration (holes in the cortex) already at 17 days of age, while glycolysis only knockdown animals develop the neurodegenerative phenotype later (38 days). $\beta$-oxidation only knockdown or control animals do not show any signs of neurodegeneration. 
Figure S3

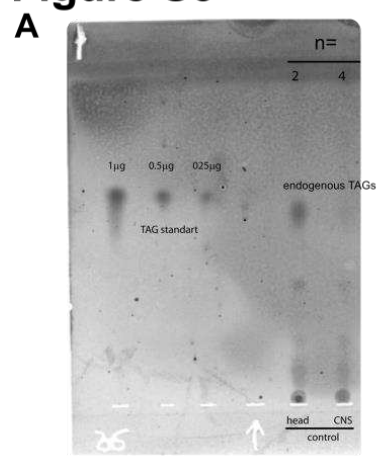

D



B

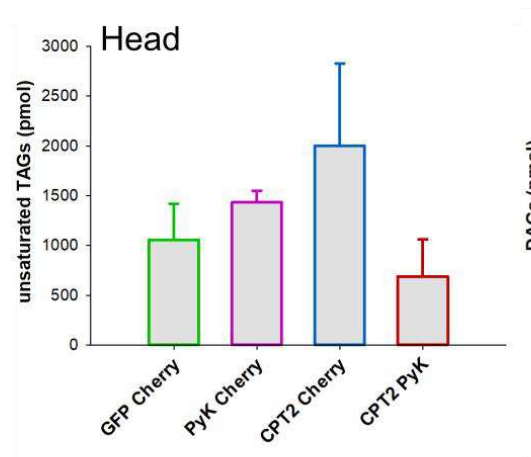

E
C

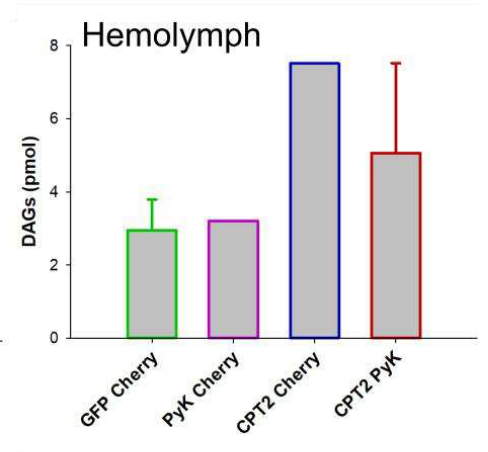

Figure S3: Glial metabolic impairment induces systemic changes in lipid mobilization. A) TLC analysis shows only a faint band of TAG in the CNS samples compared to the head samples, thus TAG levels measured in head samples can be mostly attributed to the fatbody. Sample load was normalized to polar lipids. B) Total amount of unsaturated TAGs in head samples. No significant differences are found. $\mathrm{N}=3$. Rank sum test $\mathbf{C}$ ) Total levels of DAGs measured in hemolymph samples. The measurement indicates increased DAG levels in $\beta$-oxidation knockdown as well as $\beta$-oxidation, glycolysis double knockdown animals. $\mathrm{N}=1-3$ D) Total amounts of $\mathrm{PL}$ detected in the hemolymph. Glial $\beta$-oxidation knockdown increases phospholipid levels. However, upon additional loss of glycolysis phospholipid levels are strongly reduced. $\mathrm{N}=1-3 \mathrm{E}) \mathrm{DAG}$ to $\mathrm{PL}$ ratios in the hemolymph.

\section{Supplementary files:}

Supplementary file 1: Raw data of mass spectrometry analysis of brain samples. TAG: triacylglycerol, PG: phosphatidylglycerol PE: phosphatidylethanolamine PI: phosphatidylinositol, PS: phosphatidylserine 
Supplementary file 2: Raw data of mass spectrometry analysis of head samples. TAG: triacylglycerol, PG: phosphatidylglycerol PE: phosphatidylethanolamine PI: phosphatidylinositol, PS: phosphatidylserine

Supplementary file 3: Raw data of mass spectrometry analysis of hemolymph samples. DAG: diacylglycerol, PG: phosphatidylglycerol PE: phosphatidylethanolamine PI: phosphatidylinositol, PS: phosphatidylserine 


\section{Supplementary Files}

This is a list of supplementary files associated with this preprint. Click to download.

- Supplementaryfile3.xlsx

- Supplementaryfile1.xlsx

- Supplementaryfile2.xlsx 